\title{
Dritter Phlebo-Start-up-Workshop in Aachen
}

Im Rahmen der 63. Jahrestagung in Aachen vom 08.-11. September startet nach einer Corona-Pause im Jahr 2020 nun zum dritten Mal der Hands-on „Phlebo-Start-up-Workshop“ für studentischen Nachwuchs.

Ausgerichtet ist dieser praktisch orientierte Kurs auf die Nachwuchsgeneration, auf Studenten in klinischen Semestern und angehende Ärzte im praktischen Jahr. Teilnehmen können Studenten/Studentinnen aus allen Semestern aus ganz Deutschland. Insgesamt konnte der Kurs bereits bei den ersten Durchläufen bis zu 15 Anmeldungen vorweisen. Dieses Jahr findet er in Aachen im Eurogress wieder am Mittwochnachmittag (08.09.2021) von 14:00-17:30 Uhr statt.

Im Hands-on-Kurs werden grundlegende phlebologische Themenkomplexe wie Kompressionstherapie, Duplexsonografie und Sklerotherapie vorgestellt. Hierzu wird den Teilnehmern eine duplexsonografische Untersuchung am Patienten demonstriert und die Kompressionssonografie mit anatomischen Grundlagen der Phlebologie vermittelt. Zusätzlich werden Knotentechniken sowie unterschiedliche Nahttechniken am Modell gelehrt. Insbesondere die Herstellung des Polidocanol-Schaums und das Üben am „Sklerodummy“ machen den Workshopteilnehmern in der Regel viel Spaß. Dieses Jahr wird es auch wieder eine Station zum Thema endoluminale Operationstechniken am Beispiel der endoluminalen Lasertherapie in Seldinger-Technik geben.

Darüber hinaus sind die studentischen Teilnehmer eingeladen, den Crashkurs am Mittwochvormittag zu besuchen, um die wichtigsten Grundlagen der Phlebologie zu erlernen und zu vertiefen. Spezialisiert werden kann das Wissen dann am Donnerstagvormittag im Rahmen des Weiterbildungsforums durch Vorträge der ,Jungen Phlebologen“. Zum Abschluss können Kontakte geknüpft und fachliche Gespräche am Abend bei der Phlebo-Party geführt werden. Für das leibliche Wohl wird gesorgt. Die Anreise er-

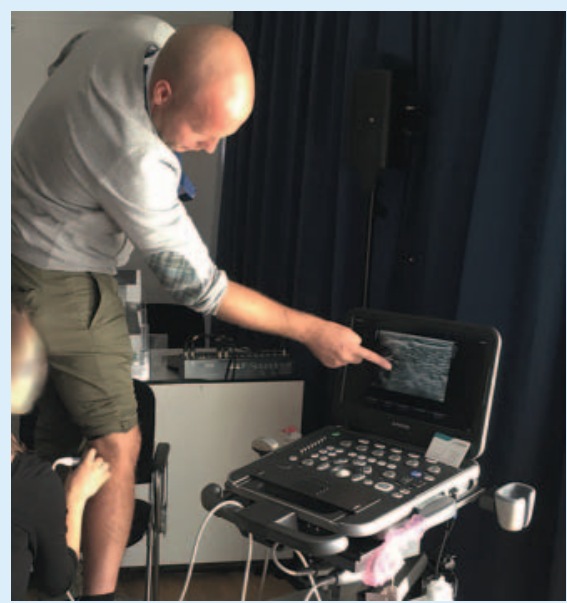

- Abb. 1 Duplexsonografie der Beinvenen durch die Kursteilnehmer. Quelle: WoitallaBruning J. Phlebo Start-Up: Erster Probedurchlauf mit Erfolg! Phlebologie 2019; 48: 132-133.

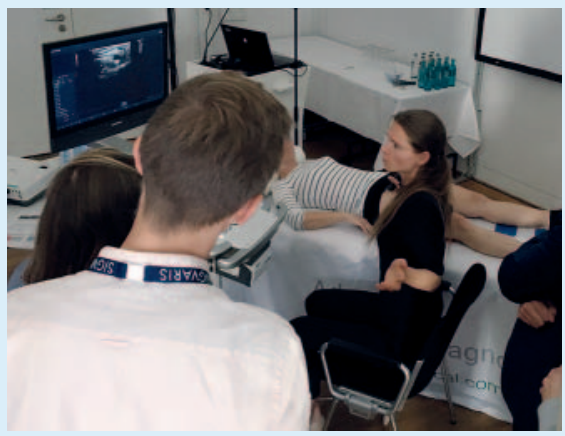

- Abb. 2 Demonstration der Kompressionssonografie. Quelle: Woitalla-Bruning J. Phlebo Start-Up: Erster Probedurchlauf mit Erfolg! Phlebologie 2019; 48: 132-133.

folgt auf eigene Faust. Finanziell unterstützt und organisiert wird der Workshop wieder durch die Deutsche Gesellschaft für Phlebologie sowie von Sigvaris.

Bei Interesse freuen wir uns über frühzeitige Anmeldungen bei begrenzter Teilnehmerzahl im Sekretariat der Deutschen Gesellschaft für Phlebologie bei Frau Pielhau, E-Mail: sekretariat@phlebology.de oder telefonisch + 49 (0)228-2871-6959.

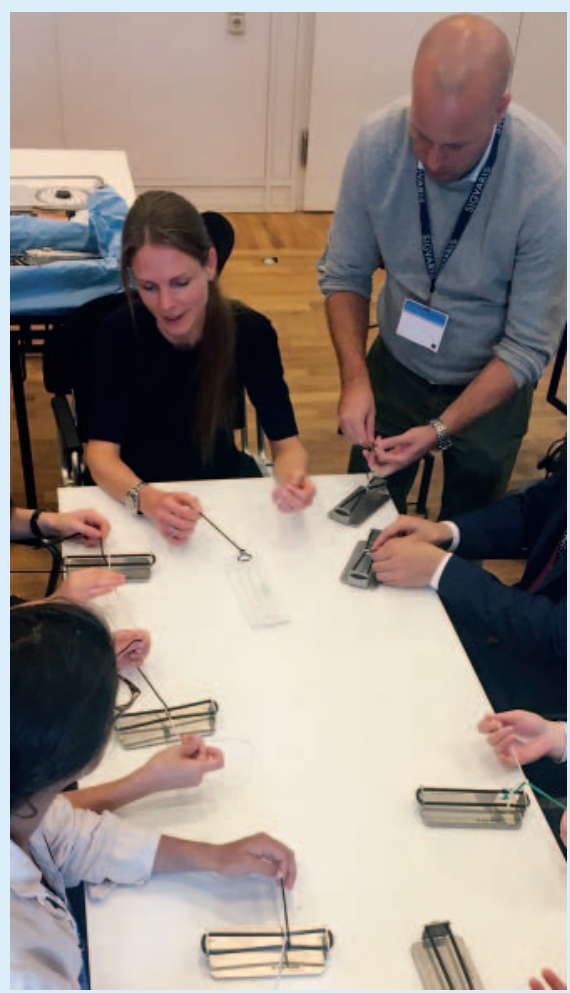

- Abb. 3 Erlernen von Knoten- und Nahttechniken am Modell. Quelle: WoitallaBruning J. Phlebo Start-Up: Erster Probedurchlauf mit Erfolg! Phlebologie 2019; 48: 132-133.

\section{Korrespondenzadresse}

Dr. Jasmin Woitalla-Bruning

Tabea-Krankenhaus, Zentrum für Venenund Dermatochirurgie, Hauttumorzentrum Hamburg am Krankenhaus Tabea Kösterbergstraße 32

22587 Hamburg

E-Mail: jwoitalla-bruning@tabea-kranken haus.de

Interessenkonflikt

Reisekostenübernahme im Rahmen von Kongressebesuchen durch die Firma Bauerfeind, Vortragshonorar durch die Firma Juzo und Sigvaris. 